\title{
Plasminogen Activator Inhibitor-1 Reduces Tissue-Type Plasminogen Activator-Dependent Fibrinolysis and Intrahepatic Hemorrhage in Experimental Acetaminophen Overdose
}

\author{
Asmita Pant, ${ }^{\dagger \dagger}$ Anna K. Kopec, ${ }^{* \dagger}$ Kevin S. Baker, ${ }^{\dagger \dagger}$ Holly Cline-Fedewa, ${ }^{*}$ Daniel A. Lawrence, ${ }^{\S}$ and James P. Luyendyk ${ }^{* \dagger}$
}

From the Departments of Pathobiology and Diagnostic Investigation* and Pharmacology and Toxicology ${ }^{\ddagger}$ and the Institute for Integrative Toxicology, ${ }^{\dagger}$ Michigan State University, East Lansing; and the Division of Cardiovascular Medicine, ${ }^{\S}$ Department of Internal Medicine, University of Michigan Medical School, Ann Arbor, Michigan

\author{
Accepted for publication \\ January $11,2018$. \\ Address correspondence to \\ James P. Luyendyk, Ph.D., \\ Department of Pathobiology \\ and Diagnostic Investigation, \\ Michigan State University, 253 \\ Food Safety and Toxicology \\ Bldg, 1129 Farm Ln, East \\ Lansing, MI 48824. E-mail: \\ luyendyk@cvm.msu.edu.
}

\begin{abstract}
Acetaminophen (APAP)-induced liver injury in mice is associated with activation of the coagulation cascade and deposition of fibrin in liver. Plasminogen activator inhibitor-1 (PAI-1) is an important physiological inhibitor of tissue-type plasminogen activator (tPA) and plays a critical role in fibrinolysis. PAI-1 expression is increased in both experimental APAP-induced liver injury and patients with acute liver failure. Prior studies have shown that PAI-1 prevents intrahepatic hemorrhage and mortality after APAP challenge, but the downstream mechanisms are not clear. We tested the hypothesis that PAI-1 limits liver-related morbidity after APAP challenge by reducing tPA-dependent fibrinolysis. Compared with APAP-challenged $(300 \mathrm{mg} / \mathrm{kg}$ ) wild-type mice, hepatic deposition of cross-linked fibrin was reduced, with intrahepatic congestion and hemorrhage increased in PAI-1-deficient mice 24 hours after APAP overdose. Administration of recombinant wild-type human PAI-1 reduced intrahepatic hemorrhage 24 hours after APAP challenge in PAI-1 $1^{-/}$mice, whereas a mutant PAI-1 lacking antiprotease function had no effect. Of interest, tPA deficiency alone did not affect APAP-induced liver damage. In contrast, fibrinolysis, intrahepatic congestion and hemorrhage, and mortality driven by PAI1 deficiency were reduced in APAP-treated $\mathrm{tPA}^{-/-} / \mathrm{PAI}-1^{-/-}$double-knockout mice. The results identify PAI-1 as a critical regulator of intrahepatic fibrinolysis in experimental liver injury. Moreover, the results suggest that the balance between PAI-1 and tPA activity is an important determinant of liver pathology after APAP overdose. (Am J Pathol 2018, 188: 1204-1212; https://doi.org/10.1016/ j.ajpath.2018.01.010)
\end{abstract}

Acetaminophen (APAP) overdose is the leading cause of acute liver failure (ALF) in the United States. ${ }^{1}$ ALF is associated with complex changes in the hemostatic system, including a rebalancing of procoagulant and anticoagulant factors that results in a retained capacity to generate thrombin. ${ }^{2,3}$ In addition, patients with ALF develop a hypofibrinolytic state, characterized by reduced plasminogen levels and increased expression of antifibrinolytic factors. ${ }^{4-6}$ Specifically, the hypofibrinolytic state in ALF patients is linked with increased expression of plasminogen activator inhibitor-1 (PAI-1), the primary inhibitor of tissue-type plasminogen activator (tPA). ${ }^{5-7}$
This rebalancing of the hemostatic system may explain the lack of spontaneous bleeding in patients with ALF despite clinical tests of coagulation, such as the prolonged prothrombin time, suggesting a bleeding tendency.

Experimental studies have also revealed connections between changes in the hemostatic system and the progression of APAP-induced liver damage in experimental

Supported by the NIH, National Institute of Diabetes and Digestive and Kidney Diseases grants R01 DK087886 and R01 DK105099 (J.P.L.).

Disclosures: None declared. 
models. For example, APAP overdose in mice is associated with activation of the coagulation cascade and deposition of fibrin clots in the liver. ${ }^{8-10}$ Like in ALF patients, plasma PAI-1 levels are increased in mice challenged with a hepatotoxic dose of APAP. ${ }^{9,11}$ Of interest, PAI-1-deficient mice develop exaggerated liver damage when challenged with hepatotoxicants, including carbon tetrachloride and APAP. ${ }^{9,11,12}$ Specifically, APAP-challenged PAI-1deficient mice develop significant intrahepatic hemorrhage and congestion and display increased mortality. ${ }^{9,11}$ However, despite strong evidence indicating that PAI-1 deficiency worsens APAP-induced liver damage, the functional impact of PAI-1 deficiency on fibrinolysis has been largely assumed, and the role of downstream PAI-1 targets, such as tPA, in APAP-induced liver injury has not been determined.

Our aim was to determine the mechanism whereby PAI-1 deficiency exaggerates liver damage after APAP challenge in mice. Using a combination of genetically modified mice with deficiencies in PAI-1 and/or tPA and mutant human PAI-1 proteins, we tested the hypothesis that PAI-1 inhibition of tPA-dependent fibrinolysis reduces liver damage after APAP overdose.

\section{Materials and Methods}

Mice

Age-matched cohorts of 10- to 15-week-old male mice were used. Wild-type C57BL/6J, PAI- $1^{-1-},{ }^{13} \mathrm{tPA}^{-1-},{ }^{14}$ and $\mathrm{PAI}-1^{-1-} / \mathrm{tPA}^{-1-}$ mice on an equivalent $\mathrm{C} 57 \mathrm{BL} / 6 \mathrm{~J}$ background were used for these studies. Wild-type mice were purchased from the Jackson Laboratory (Bar Harbor, ME). Mice were maintained in an Association for Assessment and Accreditation of Laboratory Animal Care International-accredited facility at Michigan State University (East Lansing, MI) at approximately $22^{\circ} \mathrm{C} \pm 2{ }^{\circ} \mathrm{C}$ with alternating 12-hour light/dark cycles. Mice were provided ad libitum access to reverse-osmosis purified drinking water and rodent chow diet. All animal procedures were approved by Michigan State University Institutional Animal Care and Use Committee.

\section{Treatment Paradigms and Tissue Collection}

Mice were fasted overnight (ie, approximately 16 hours) before administration of $300 \mathrm{mg} / \mathrm{kg}$ APAP (at $30 \mathrm{~mL} / \mathrm{kg}$ body weight) or vehicle via i.p. injection. Food was returned immediately after APAP injection. In some experiments, the mice were given injections of $100 \mu \mathrm{g}$ recombinant human PAI-1 protein with intact antiprotease function (PAI- $1_{C}$ ) with improved half-life, ${ }^{15}$ recombinant PAI-1 protein lacking a functional antiprotease domain $\left(\mathrm{PAI}-1_{\mathrm{RR}}\right),{ }^{16}$ or vehicle (sterile saline solution), 1 hour prior and 8 hours after APAP injection. At 6 or 24 hours after APAP administration, mice were anesthetized using isoflurane, and blood was collected from the caudal vena cava into a syringe containing sodium citrate $(0.38 \%$ final) for the collection of plasma. The liver was excised and washed in phosphate-buffered saline. The entire left lateral lobe was fixed in $10 \%$ neutral-buffered formalin for approximately 96 hours and processed for routine histopathological analysis. Small sections of liver from different lobes were snap frozen in liquid nitrogen and saved for other analyses, including capillary-based Western blot analysis.

\section{Histopathology, Immunohistochemistry, and Biochemical Analysis}

All immunohistochemical staining was performed by the Michigan State University Investigative Histopathology Laboratory, a division of Human Pathology. Paraffinembedded livers were cut into sections (5 $\mu \mathrm{m}$ thick) and stained with hematoxylin and eosin. For quantification of hepatic necrosis and congestion/hemorrhage, a Virtual Slide System VS110 (Olympus, Tokyo, Japan) was used to capture images of stained liver sections. The extent of APAP-induced hepatocellular necrosis and congestion/ hemorrhage was quantified in the digitized slides using the STEPanizer software version 1 (http://www. stepanizer.com). ${ }^{17}$ At least 10 representative low-magnification hematoxylin and eosin-stained liver sections from each animal were examined, and the results were expressed as a percentage of necrosis or congestion/hemorrhage relative to the total area examined. Immunofluorescence staining for fibrin(ogen) was performed as described previously. ${ }^{18}$ Briefly, rabbit anti-human fibrinogen antibody (A0080; Dako, Carpinteria, CA) was used as the primary antibody and was detected by goat anti-rabbit IgG conjugated to Alexa Fluor 594 (A11008; Invitrogen, Carlsbad, CA) and visualized by fluorescent microscopy. Alanine aminotransferase (ALT) activity was measured using a commercially available reagent (Infinity ALT/GPT; Thermo Fisher Scientific, Waltham, MA). Plasma D-dimer levels were measured using the Asserachrom D-Di kit (Diagnostica Stago, Inc., Parsippany, NJ), according to the manufacturer's protocol. Plasma thrombin-antithrombin levels were determined using commercial reagents (Siemens Healthcare Diagnostics, Tarrytown, NY).

\section{Detection of Intrahepatic HMW Fibrin(ogen) by Capillary Western Blot Analysis}

Intrahepatic levels of urea-insoluble (ie, cross-linked ${ }^{19,20}$ ) high-molecular-weight (HMW) fibrin(ogen) was determined, as described previously, ${ }^{21}$ based on protocols adapted for determining fibrin content in venous thrombi. ${ }^{22}$ Briefly, $70 \mathrm{mg}$ of snap-frozen liver was homogenized in 1 $\mathrm{mL}$ of solution containing $8 \mathrm{~mol} / \mathrm{L}$ urea, $40 \mathrm{mmol} / \mathrm{L}$ dithiothreitol, and $12.5 \mathrm{mmol} / \mathrm{L}$ EDTA, rotated end-overend at $60^{\circ} \mathrm{C}$ for 1 hour, and then incubated at $60^{\circ} \mathrm{C}$ overnight (approximately 16 hours). The samples were then spun at $10,000 \times g$ for 5 minutes and resolved on Wes 66 to 
A

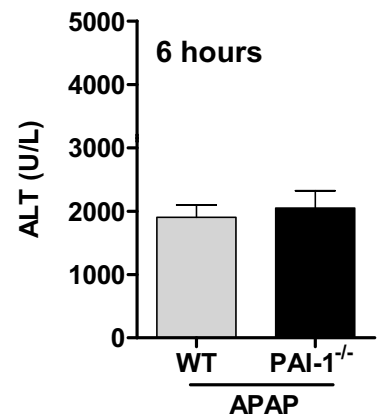

C

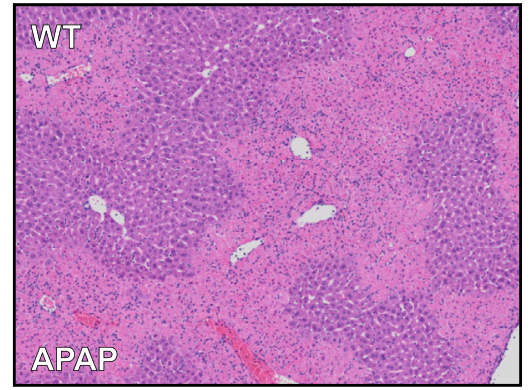

D

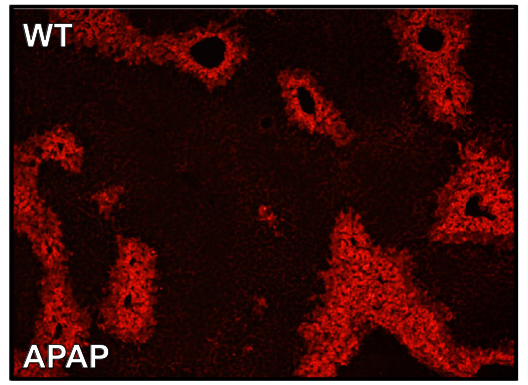

E

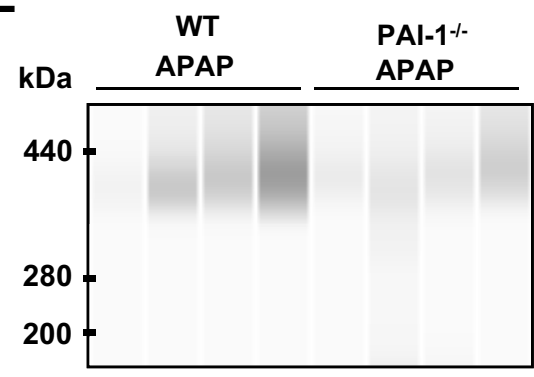

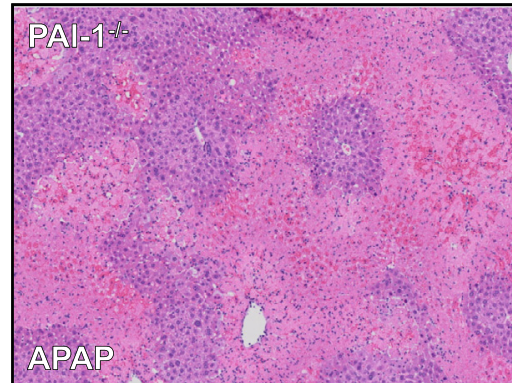

B

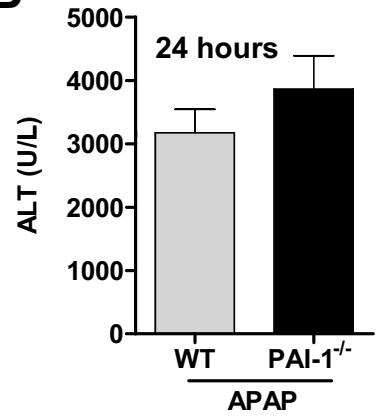

APAP

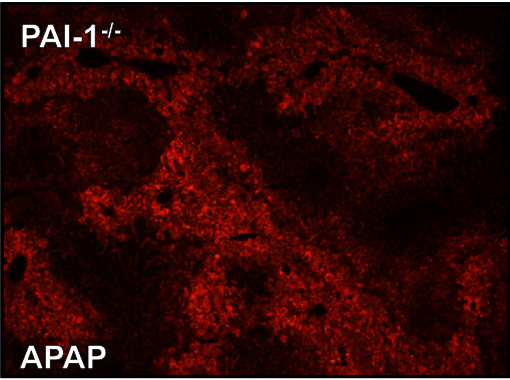

$\mathbf{F}$

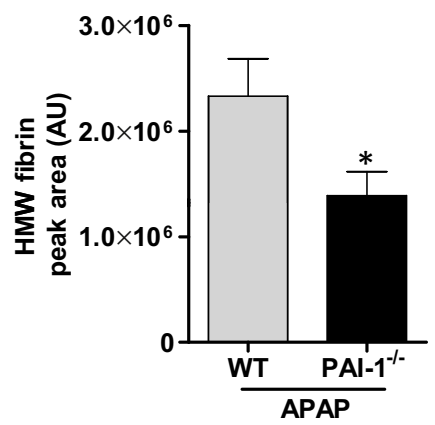

Figure 1 Hepatic injury and fibrin(ogen) levels in PAI-1 ${ }^{-1-}$ mice after acetaminophen (APAP) overdose. Male wild-type (WT) and PAI- $1^{-/-}$mice were given $300 \mathrm{mg} / \mathrm{kg}$ APAP (i.p.). Liver and plasma were collected at 6 or 24 hours after APAP challenge. Plasma alanine aminotransferase (ALT) activity at 6 hours (A) and 24 hours (B), representative photomicrographs of hematoxylin and eosin-stained liver sections at 24 hours (C), representative photomicrographs showing immunofluorescence labeling of fibrin(ogen) (red) at 24 hours (D), representative image showing hepatic levels of fibrin(ogen) species detected using capillary Western blot at 24 hours $(\mathbf{E})$, and quantification of the corresponding high-molecular-weight (HMW; >350 kDa) fibrin bands (F), as described in Materials and Methods. Data are expressed as means $\pm \operatorname{SEM}(\mathbf{A}, \mathbf{B}$, and $\mathbf{F})$. $n=9-12$ mice per group (A, $\mathbf{B}$, and $\mathbf{F}) .{ }^{*} P<0.05$ (significantly different from APAP-challenged WT mice). Original magnification, $\times 100$ (C and D). $\mathrm{AU}$, arbitrary unit.
440-kDa 25-capillary gels (Protein Simple, San Jose, CA). Fibrin(ogen) was detected with polyclonal rabbit antihuman fibrinogen antibody (Dako), using Wes Rabbit Master Kit, according to the manufacturer's protocol (Protein Simple). Peaks corresponding to HMW (>350 kDa) fibrin(ogen) were quantified using the Compass software version 3.1.7 (Protein Simple).

\section{Statistical Analysis}

Comparison of two groups was performed using $t$-test. Comparison of the three groups was done using one-way analysis of variance and the Student-Newman-Keuls post hoc test. Differences were considered significant when $P<0.05$. 


\section{Results}

Effect of PAI-1 Deficiency on Hepatic Injury and Fibrin Deposits in APAP-Challenged Mice

In agreement with previous studies, plasma ALT activity was similar in APAP-challenged PAI- $1^{-1-}$ mice at 6 hours compared with APAP-challenged wild-type mice ${ }^{11}$ (Figure 1A). Plasma ALT activity and centrilobular necrosis were modestly increased at 24 hours in APAP-challenged PAI- $1^{-/-}$mice compared with APAP-challenged wild-type mice (Figure 1, B and C). Of interest, a marked increase in intrahepatic hemorrhage was observed in APAP-challenged PAI- $1^{-1-}$ mice compared with APAP-challenged wild-type mice at 24 hours (Figure 1C). Fibrin(ogen) deposits in livers of APAP-challenged wild-type mice were largely restricted to areas of hepatic necrosis (Figure 1D). Fibrin(ogen) deposits in livers of APAP-challenged PAI-1 ${ }^{-1-}$ mice were similarly distributed within necrotic lesions, but were more diffuse in appearance (Figure 1D). Prior studies have speculated that PAI-1 stabilizes intrahepatic fibrin in livers of APAPchallenged mice, ${ }^{11}$ but this has not been formally demonstrated. To isolate the specific effect of PAI-1 deficiency on deposition of HMW cross-linked fibrin polymer deposited in liver, capillary gel-based Western blot analysis was used. More important, hepatic levels of urea-insoluble HMW fibrin were significantly reduced in livers of APAPchallenged $\mathrm{PAI}-1^{-1-}$ mice compared with APAPchallenged wild-type mice (Figure 1, E and F).

\section{PAI-1 Antiprotease Activity Reduces Hepatic Congestion/Hemorrhage in APAP-Challenged Mice}

Through its antiprotease domain, PAI-1 inhibits conversion of plasminogen to plasmin by the plasminogen activators tPA and urokinase plasminogen activator (uPA). To determine whether this specific function of PAI-1 was central to its inhibition of intrahepatic hemorrhage after APAP challenge, gain-of-function studies were performed in APAP-challenged PAI- $1^{-1-}$ mice. Specifically, mice were given either a stable PAI- $1_{\mathrm{C}}$ or stable PAI- $1_{\mathrm{RR}}$. A rapid and significant increase in PAI-1 plasma is evident at 6 hours after APAP overdose in mice. ${ }^{8}$ To reconstitute PAI-1 at a time that mimics the timing of this increase, APAPchallenged $\mathrm{PAI}-1^{-/-}$mice were given either saline or recombinant PAI-1 (100 $\mu \mathrm{g} / \mathrm{mouse}), 1$ hour prior and 8 hours after APAP challenge. PAI- $1_{C}$ did not significantly reduce hepatocellular injury 24 hours after APAP challenge in PAI- $1^{-1-}$ mice, indicated by plasma ALT activity and
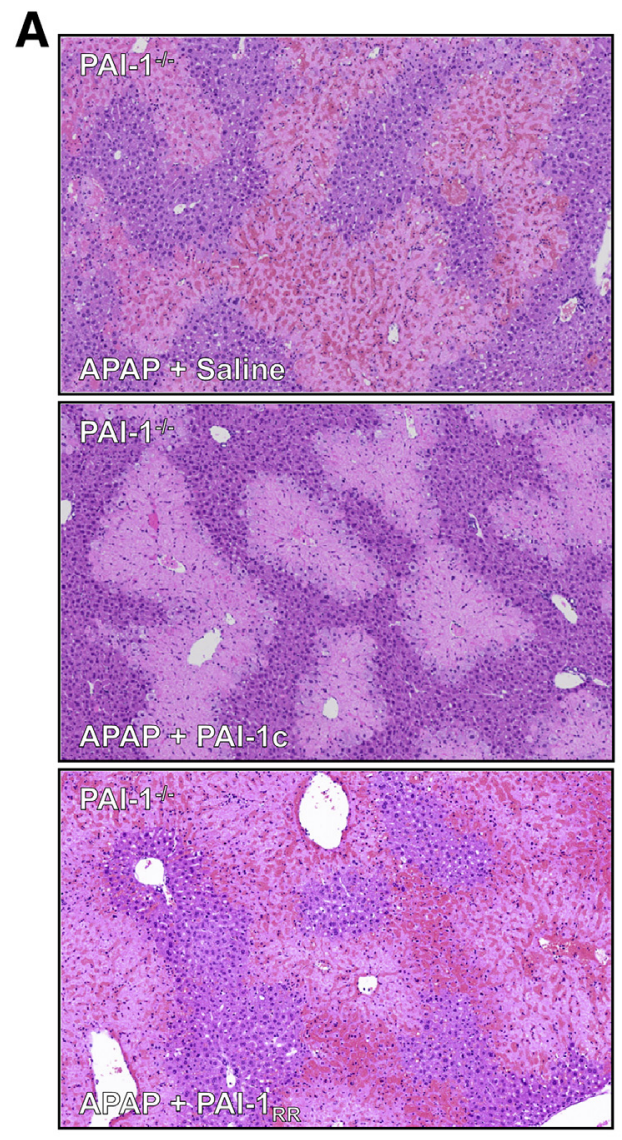
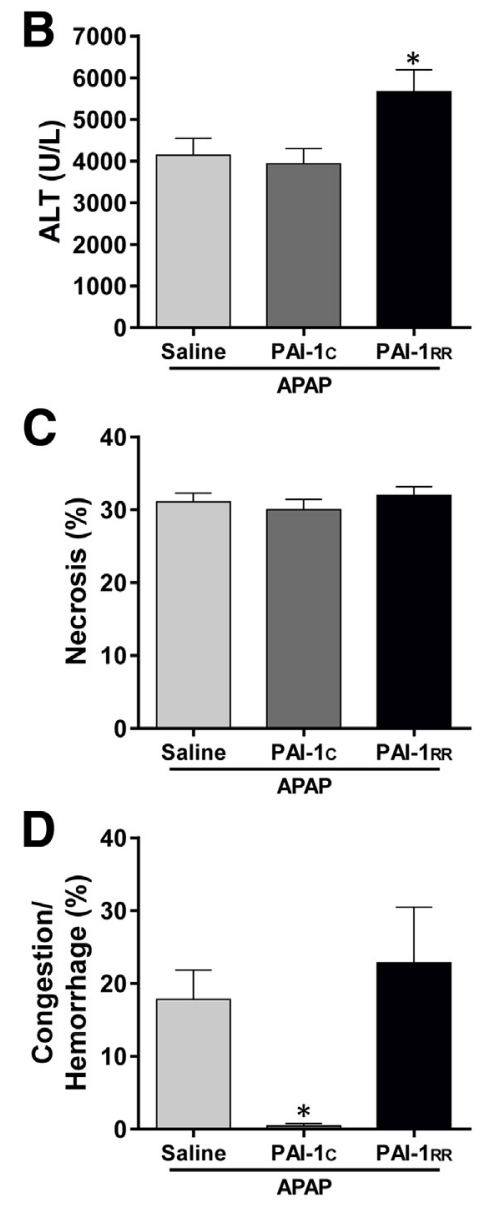

Figure 2 Mutant PAI-1 lacking antiprotease activity has no effect on acetaminophen (APAP)induced hepatic injury in PAI-1 $1^{-/-}$mice. Male PAI-1-deficient mice were injected with sterile saline (vehicle), recombinant stable wild-type human PAI-1 (PAI-1 1 ), or mutant PAI-1 protein lacking antiprotease activity (PAI- $\left.1_{\mathrm{RR}}\right)$ at 1 hour prior and 8 hours after $300 \mathrm{mg} / \mathrm{kg}$ APAP (i.p.) treatment. Liver and plasma were collected 24 hours after APAP challenge. Representative photomicrographs of hematoxylin and eosin-stained liver sections (A), plasma alanine aminotransferase (ALT) activity (B), the area of centrilobular necrosis in liver sections (C), and the area of congestion/hemorrhage in liver sections (D) were determined, as described in Materials and Methods. Data are expressed as means \pm SEM (B-D). $n=6$ (saline-treated group; B-D); $n=8$ (groups that were given back either PAI- $1_{C}$ or PAI- $\left.1_{R R} ; \mathbf{B}-\mathbf{D}\right)$. ${ }^{*} P<0.05$ (significantly different from salinetreated APAP-challenged PAI- $1^{-/-}$mice). Original magnification, $\times 100(A)$. 
area of hepatocellular necrosis (Figure 2, A-C). Of interest, plasma ALT activity was significantly increased by PAI- $1_{R R}$ administration, although this was not coupled to a change in necrosis (Figure 2, B and C). Marked intrahepatic congestion with frequent hemorrhage is the primary lesion that distinguishes PAI- $1^{-1-}$ mice from wild-type mice at this time point after APAP challenge. ${ }^{9}$ Although there was no effect of PAI- $1_{C}$ on necrosis, administration of PAI- $1_{C}$ to APAP-challenged PAI- $1^{-1-}$ mice significantly reduced congestion/hemorrhage (Figure 2, A and D). In contrast, this protective effect was completely absent in mice given PAI- $1_{\text {RR }}$ (Figure 2, A and D). Plasma levels of D-dimer, a fibrin degradation product and indicator of plasminmediated fibrinolytic activity, were also assessed in APAP-challenged PAI-1 ${ }^{-/-}$mice. Compared with saline-treated mice $(52.4 \pm 4.87 \mathrm{ng} / \mathrm{mL})$, plasma D-dimer level tended to decrease in PAI- $1_{\mathrm{C}}-$ treated mice $(30.4 \pm$ $9.4 \mathrm{ng} / \mathrm{mL}$ ), but not in PAI- $1_{\mathrm{RR}}-$ treated mice $(77.6 \pm$ $9.9 \mathrm{ng} / \mathrm{mL}$ ), suggesting reduced fibrinolysis in response to PAI- $1_{C}$ administration. The results indicate that PAI-1 antiprotease function is central to its protective effects in experimental APAP overdose.

\section{Intrahepatic Congestion/Hemorrhage after APAP} Overdose in PAI-1 ${ }^{-/}$Mice Is Driven by Exaggerated tPA-Dependent Fibrinolysis

Because the protective effects of PAI-1 required antiprotease function, the role of tPA in APAP-induced liver injury was determined. Of interest, APAP-induced liver injury was virtually identical in $\mathrm{tPA}^{-/-}$mice compared with wild-type mice, indicated by centrilobular necrosis of similar severity, absence of severe congestion/hemorrhage, and comparable plasma ALT activity (Figure 3). Thus, it appears that when PAI-1 is available at sufficient levels, tPA does not play a critical role in APAP-induced liver injury. There was no evidence of compensatory induction of uPA in APAP-treated $\mathrm{PAA}^{-1-}$ mice, as determined by plasminogen zymography (data not shown). A previous study found that administration of tenecteplase, a recombinant tPA less sensitive to inhibition by PAI- $1,{ }^{23}$ increased congestion/hemorrhage in livers of APAPchallenged wild-type mice. ${ }^{9}$ To determine whether exaggerated tPA activity increases fibrinolysis and liver injury in PAI- $1^{-l-}$ mice, mice lacking both PAI-1 and tPA $\left(\mathrm{PAI}-1^{-1-} / \mathrm{tPA}^{-1-}\right.$ mice) were used.

Prior studies have left the exact role of PAI-1 in fibrinolysis in the context of APAP-induced liver injury uninvestigated. Thus, plasma D-dimer levels were measured, which increased significantly in APAP-challenged PAI-1 ${ }^{-1-}$ mice compared with APAP-challenged wild-type mice (Figure 4A). This increase in fibrinolysis was coincident with a reduction in hepatic urea-insoluble HMW fibrin deposits (Figure 4, C and D). Of note, tPA deficiency completely prevented exaggerated fibrinolysis in APAPchallenged PAI- $1^{-1-}$ mice, as indicated by D-dimer levels in $\mathrm{PAI}-1^{-/-} / \mathrm{tPA}^{-1-}$ mice, similar to APAP-challenged wild-type mice (Figure 4A), and by preservation of hepatic HMW fibrin deposition (Figure 4, C and D). Notably, plasma thrombin-antithrombin, an indicator of coagulation cascade activation, was similar in mice of each genotype (Figure 4B). This indicates that changes in D-dimer and hepatic fibrin were driven by fibrinolysis and not upstream effects of coagulation. Collectively, the results suggest that in the absence of its inhibitor PAI-1, exaggerated tPAdependent fibrinolysis is evident after APAP challenge.

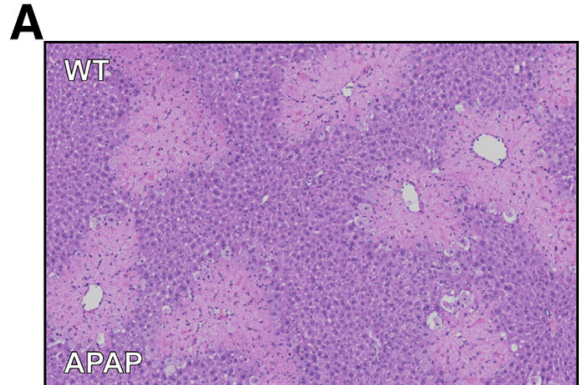

B

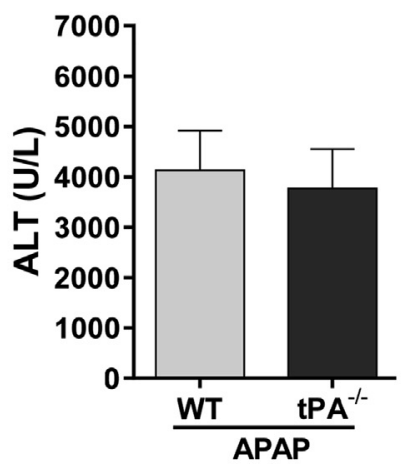

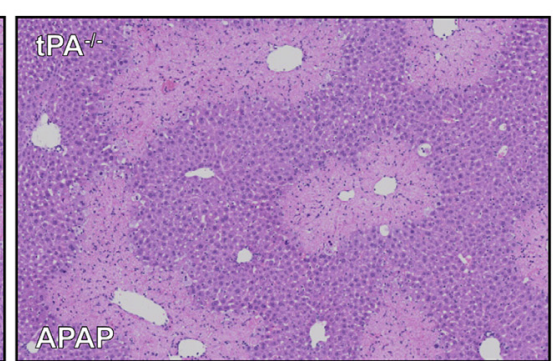

C

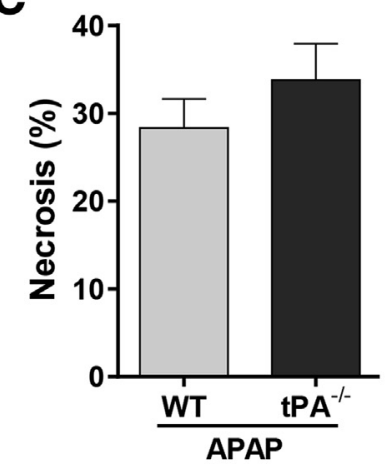

Figure 3 Hepatic injury in $\mathrm{tPA}^{-/-}$mice after acetaminophen (APAP) overdose. Male wild-type (WT) and $\mathrm{tPA}^{-/-}$mice were injected with 300 $\mathrm{mg} / \mathrm{kg}$ APAP (i.p.), and liver injury was examined 24 hours later. Representative photomicrographs of hematoxylin and eosin-stained liver sections (A), plasma alanine aminotransferase (ALT) levels (B), and the area of centrilobular necrosis in liver sections (C) were determined, as described in Materials and Methods. Data are expressed as means \pm SEM (B and $\mathbf{C}) \cdot n=6-9$ mice per group $(\mathbf{B}$ and $\mathbf{C})$. Original magnification, $\times 100(\mathbf{A})$. 

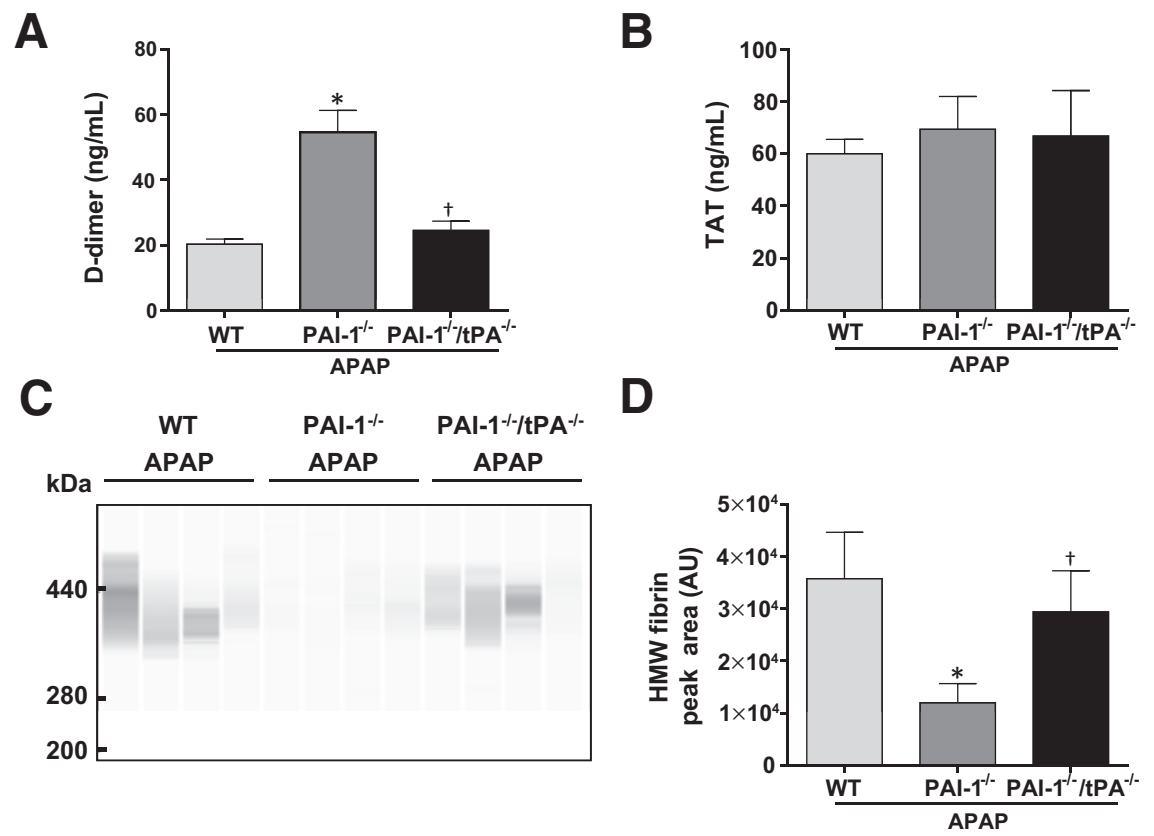

Figure 4 Hepatic fibrinolysis in PAI-1 ${ }^{-/-} / \mathrm{tPA}^{-/-}$ mice after acetaminophen (APAP) overdose. Male wildtype (WT), PAI- $1^{-/-}$, and PAI- $1^{-/-} / \mathrm{tPA}^{-/-}$mice were injected with $300 \mathrm{mg} / \mathrm{kg}$ APAP (i.p.). Liver injury and fibrinolysis were examined 24 hours after APAP challenge. Plasma D-dimer levels (A), plasma thrombin-antithrombin (TAT) levels (B), representative image showing hepatic levels of fibrin(ogen) species detected using capillary Western blot (C), and quantification of the corresponding high-molecular-weight (HMW; $>300 \mathrm{kDa}$ ) fibrin bands (D) were determined, as described in Materials and Methods. Data are expressed as means $\pm \operatorname{SEM}(\mathbf{A}, \mathbf{B}$, and D). $n=7-13$ mice per group (A, B, and $\mathbf{D})$. ${ }^{*} P<0.05$ (significantly different from the WT APAP group); ${ }^{\dagger} P<0.05$ (significantly different from the PAI- $1^{-/-}$APAP group). AU, arbitrary unit.

Next, the effect of tPA deficiency was determined on liver pathology in PAI-1-deficient mice. As anticipated, hepatic injury and, most notably, congestion/hemorrhage were significantly increased in APAP-challenged PAI- $1^{-/-}$mice compared with APAP-challenged wild-type mice (Figure 5). Of interest, tPA deficiency significantly reduced liver damage caused by PAI- 1 deficiency, denoted by decreased centrilobular necrosis and plasma ALT activity (Figure 5, A-C), although changes in ALT did not achieve statistical significance (Figure 5B). There was also a markedly diminished area of congestion/hemorrhage in APAPchallenged PAI- $1^{-/-} / \mathrm{tPA}^{-l-}$ mice compared with PAI- $1^{-1-}$ mice (Figure 5, A and D). Indeed, liver histology in $\mathrm{PAI}^{-1}{ }^{-1-} / \mathrm{tPA}^{-1-}$ mice closely resembled wild-type mice (Figure 5A). Importance of these changes to overall outcome was illustrated by approximately $30 \%$ mortality in APAPchallenged PAI- $1^{-1-}$ mice and a reduction in mortality in PAI- $1^{-l-} / \mathrm{tPA}^{-1-}$ mice $(10 \%, P=0.08)$. The results suggest that exaggerated tPA-mediated fibrinolysis exacerbates APAP-induced liver damage in the absence of PAI-1.

\section{Discussion}

Plasma PAI-1 levels are significantly increased in patients with ALF, and this is recapitulated in experimental models of liver damage. , $5,9,11,12$ In ALF patients, the increase in PAI-1 levels is thought to drive a hypofibrinolytic state. ${ }^{4}$ Previous studies have described increased liver damage in the absence of PAI-1 in experimental mice, ${ }^{9}$ although the underlying mechanisms were largely unknown and the precise impact of PAI-1 on fibrinolytic activity had not been explored. Herein, we demonstrate a critical role of PAI-1 in regulating in vivo tPA-dependent fibrinolysis after APAP overdose. Rescue of PAI- $1^{-1-}$ mice with PAI- $1_{\mathrm{C}}$ links exaggerated APAP-induced liver injury in these mice to the loss of PAI-1. Moreover, experiments using mutant PAI-1 lacking antiprotease function and double-deficient PAI-1/ tPA mice demonstrate that uncontrolled tPA-dependent fibrinolysis in the absence of PAI-1 accounts for the liver phenotype observed in APAP-challenged PAI- ${ }^{-/-}$mice. Collectively, the results are the first to definitively link increased fibrinolysis with exaggerated liver damage after APAP challenge.

Plasma fibrinogen levels are reduced in patients with ALF and in mice after APAP overdose. ${ }^{24,25}$ In mice, this reduction in plasma fibrinogen is coupled to thrombindriven deposition of fibrin(ogen) within the intrahepatic necrotic regions. ${ }^{8}$ Although it was previously hypothesized that fibrin(ogen) deposits in liver contributed to the progression of liver injury, ${ }^{8}$ a recent study has replaced this assumption with experimental evidence indicating a role for fibrin(ogen) in liver repair. ${ }^{21}$ Cross-linked fibrin polymers can engage leukocyte integrin $\alpha_{M} \beta_{2}$ and direct inflammatory functions of macrophages in multiple disease states. ${ }^{26}$ This specific function of fibrin(ogen) was critical for macrophage activation, clearance of necrotic material, and stimulation of hepatocyte proliferation after APAP overdose. ${ }^{21}$ Hepatic cross-linked fibrin deposits were reduced in APAP-challenged PAI- $1^{-1-}$ mice, and this was coupled to an increase in plasma D-dimer. Notably, these changes were largely corrected by the absence of tPA. Thus, accelerated degradation of fibrin in livers of PAI- $1^{-1-}$ mice may explain the exaggerated injury after APAP challenge. However, both tPA and plasmin have multiple substrates, and tPA can also affect disease pathogenesis through plasminogen-independent mechanisms. ${ }^{27-29}$ Therefore, the 


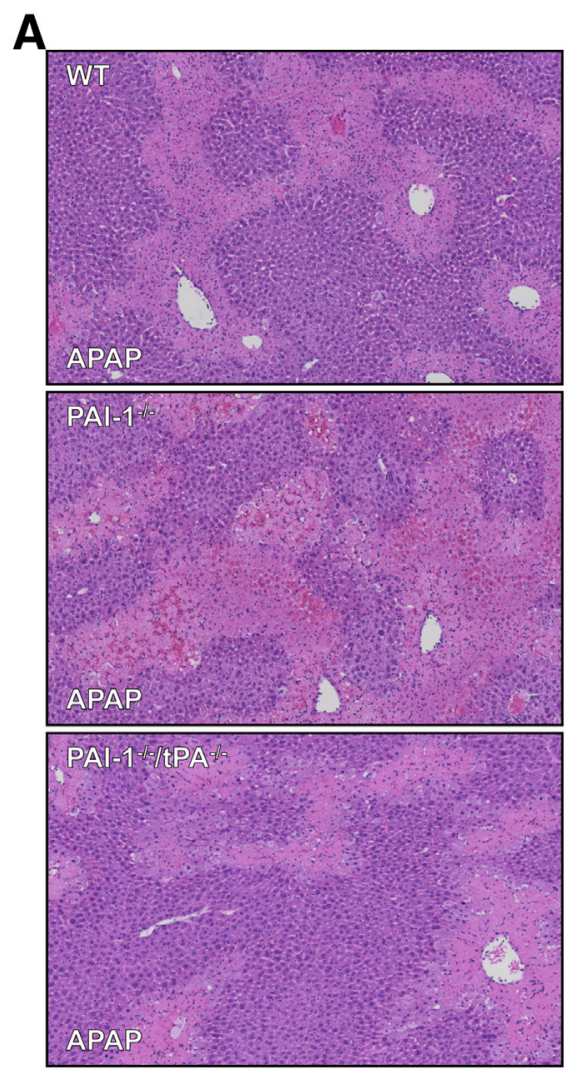

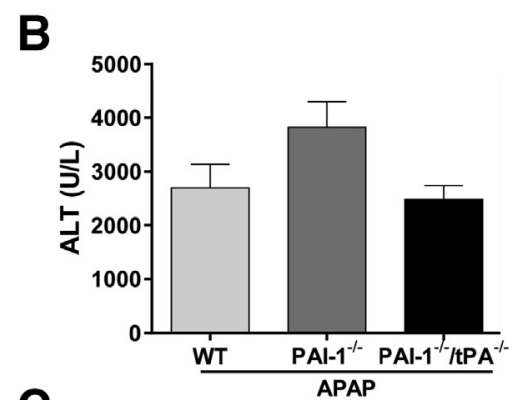

C

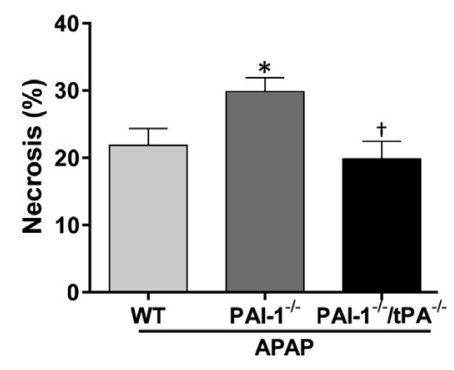

D

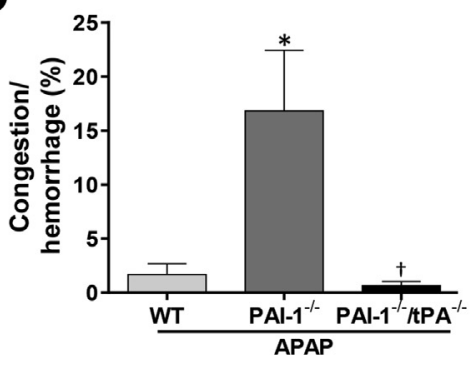

Figure 5 Hepatic injury in PAI- $1^{-/-} / \mathrm{tPA}^{-/-}$ mice after acetaminophen (APAP) overdose. Male wild-type (WT), PAI- $1^{-/-}$, and PAI-1 ${ }^{-/-} / \mathrm{tPA}^{-/-}$ mice were injected with $300 \mathrm{mg} / \mathrm{kg}$ APAP (i.p.). Liver injury and fibrinolysis were examined 24 hours after APAP challenge. Representative photomicrographs of hematoxylin and eosin-stained liver sections (A), plasma alanine aminotransferase (ALT) activity (B), centrilobular necrosis (C), and area of congestion/hemorrhage (D) were determined, as described in Materials and Methods. Data are expressed as means \pm SEM (B-D). $n=7-13$ mice per group (B-D). ${ }^{*} P<0.05$ (significantly different from the WT APAP group); ${ }^{\dagger} P<0.05$ (significantly different from the PAI- $1^{-/-}$APAP group). Original magnification, $\times 100(\mathbf{A})$. possibility that there is a fibrin(ogen)-independent mechanism whereby increased tPA activity drives APAP hepatotoxicity cannot be excluded. Also, a role for uPA activity in fibrinolysis in APAP-challenged PAI- $1^{-/-}$mice cannot be excluded. Prior studies demonstrate a role for uPA and uPA receptor in intrahepatic fibrinolysis, albeit in the normal liver. ${ }^{30}$ It is possible that tPA deficiency reduces liver injury because it reduces total hepatic plasminogen activator function. Additional studies will be required to determine the precise role of UPA in APAP-induced liver injury.

Exacerbation of hepatic pathology in PAI-1 $1^{-/-}$mice, particularly congestion and hemorrhage, was not evident in $\mathrm{PAI}-1^{-1-} / \mathrm{tPA}^{-1-}$ mice. Likewise, PAI- $1_{\mathrm{RR}}$ failed to reduce liver injury in APAP-challenged PAI-1 $1^{-/}$mice. These studies indicate that in the absence of PAI-1, tPA activity exacerbates APAP-induced liver injury. Surprisingly, however, tPA deficiency had no effect on liver injury in APAP-challenged wild-type mice. This indicates that a pathologic role for tPA in APAP-induced liver injury is revealed only in conditions where PAI-1 levels are reduced; when PAI-1 levels are sufficient, tPA is not central to the mechanism whereby APAP injures the liver. Of note, however, administration of tenecteplase, a recombinant PAI-1 resistant form of human tPA, significantly increased liver injury in APAP-challenged wild-type mice, and liver pathology resembled that of APAP-challenged $\mathrm{PAI}-1^{-1-}$ mice. ${ }^{9}$ Collectively, these studies imply that PAI-1 plays a critical role in limiting excessive fibrinolytic activity in the APAP-injured liver and that excess tPA activity worsens APAP-induced liver injury. In experimental settings of APAP challenge, failed liver repair is often associated with excess congestion/hemorrhage. ${ }^{11,21,31}$ However, the precise contribution of this lesion in acute liver injury is not clear. Congestion/hemorrhage appeared to be disconnected from severity of hepatic necrosis, but interestingly, a reduction in the severity of this lesion was associated with an apparent decrease in mortality.

PAI-1 displays both protective and damaging roles in experimental settings of liver disease, and its contribution certainly seems to be context dependent. ${ }^{11,12,30,32-35}$ There is ample evidence that PAI-1 deficiency exacerbates APAP-induced liver damage in experimental mice; however, the clinical implications of this observation are not yet clear. For example, a few clinical studies observed that blood levels of PAI-1 are increased in ALF patients, analogous to patients with cirrhosis. ${ }^{4,5}$ One study reported that ALF patients developed a hypofibrinolytic state, indicated by a reduced rate of tPA-dependent clot lysis ex vivo. ${ }^{4}$ To date, however, an association between PAI-1 levels, or other measures of fibrinolysis, with outcome or measures of injury in ALF patients has not been identified. It is possible that a failure to express sufficient PAI-1 may exacerbate tPA activity, permitting excess intrahepatic 
fibrinolysis, congestion, and hemorrhage. This is a challenging hypothesis to test, particularly because measures of fibrinolysis, such as D-dimer, ${ }^{36}$ are also affected by the rate of thrombin-mediated fibrin deposition, which, in turn, is likely driven by liver tissue factor-mediated coagulation. ${ }^{9}$ Moreover, it is possible that changes in plasma markers of fibrinolysis may not directly reflect differences in intrahepatic fibrinolytic activity. Regardless, the observation that the balance between PAI-1 and tPA activity critically regulates liver damage in experimental mice suggests that future studies examining the association between PAI-1 levels, as well as measures of fibrinolysis (eg, tPA and D-dimer), in a large cohort of ALF patients would be valuable.

In summary, these studies identified that PAI-1 deficiency drives intrahepatic congestion/hemorrhage and mortality after APAP overdose by a mechanism linked to increased tPA-dependent fibrinolysis. This suggests that the balance of profibrinolytic and antifibrinolytic factors is a critical determinant of the extent of experimental APAPinduced liver injury. Acknowledging the limitation of experimental mice with complete PAI-1 deficiency, it is possible that reduced PAI-1 levels in patients with ALF may also affect liver injury pathogenesis. Insofar as plasminogen deficiency or pharmacologic inhibition reduced experimental APAP hepatotoxicity in mice, ${ }^{9}$ pharmacologic inhibition of this pathway with approved drugs, such as tranexamic acid, may be beneficial in APAP overdose patients. Collectively, these observations inform clinical studies in ALF patients by suggesting the possibility that changes in fibrinolysis may affect not only the risk of bleeding or thrombosis, but also fundamentally affect liver injury pathogenesis, and perhaps even repair of the injured liver after APAP overdose.

\section{Acknowledgments}

We thank Dr. Lauren G.P. Hardy for technical assistance.

A.P., A.K.K., K.S.B., H.C.-F., D.A.L., and J.P.L. designed and conducted the experiments; A.P., A.K.K., and J.P.L. analyzed the data; A.P. and J.P.L. wrote the manuscript; and A.P., A.K.K., K.S.B., H.C.-F., D.A.L., and J.P.L. approved the final manuscript.

\section{References}

1. Lee WM: Acetaminophen-related acute liver failure in the United States. Hepatol Res 2008, 38 Suppl 1:S3-S8

2. Lisman T, Porte RJ: Rebalanced hemostasis in patients with liver disease: evidence and clinical consequences. Blood 2010, 116: 878-885

3. Lisman T, Stravitz RT: Rebalanced hemostasis in patients with acute liver failure. Semin Thromb Hemost 2015, 41:468-473

4. Lisman T, Bakhtiari K, Adelmeijer J, Meijers JC, Porte RJ, Stravitz RT: Intact thrombin generation and decreased fibrinolytic capacity in patients with acute liver injury or acute liver failure. J Thromb Haemost 2012, 10:1312-1319
5. Pernambuco JR, Langley PG, Hughes RD, Izumi S, Williams R: Activation of the fibrinolytic system in patients with fulminant liver failure. Hepatology 1993, 18:1350-1356

6. De Gasperi A, Corti A, Mazza E, Prosperi M, Amici O, Bettinelli L: Acute liver failure: managing coagulopathy and the bleeding diathesis. Transplant Proc 2009, 41:1256-1259

7. Cale JM, Lawrence DA: Structure-function relationships of plasminogen activator inhibitor-1 and its potential as a therapeutic agent. Curr Drug Targets 2007, 8:971-981

8. Ganey PE, Luyendyk JP, Newport SW, Eagle TM, Maddox JF, Mackman N, Roth RA: Role of the coagulation system in acetaminophen-induced hepatotoxicity in mice. Hepatology 2007, 46: $1177-1186$

9. Sullivan BP, Kassel KM, Jone A, Flick MJ, Luyendyk JP: Fibrin(ogen)independent role of plasminogen activators in acetaminophen-induced liver injury. Am J Pathol 2012, 180:2321-2329

10. Sullivan BP, Kopec AK, Joshi N, Cline H, Brown JA, Bishop SC, Kassel KM, Rockwell C, Mackman N, Luyendyk JP: Hepatocyte tissue factor activates the coagulation cascade in mice. Blood 2013, 121: $1868-1874$

11. Bajt ML, Yan HM, Farhood A, Jaeschke H: Plasminogen activator inhibitor-1 limits liver injury and facilitates regeneration after acetaminophen overdose. Toxicol Sci 2008, 104:419-427

12. von Montfort C, Beier JI, Kaiser JP, Guo L, Joshi-Barve S, Pritchard MT, States JC, Arteel GE: PAI-1 plays a protective role in CCl4-induced hepatic fibrosis in mice: role of hepatocyte division. Am J Physiol Gastrointest Liver Physiol 2010, 298:G657-G666

13. Carmeliet P, Kieckens L, Schoonjans L, Ream B, van Nuffelen A, Prendergast G, Cole M, Bronson R, Collen D, Mulligan RC: Plasminogen activator inhibitor-1 gene-deficient mice, I: generation by homologous recombination and characterization. J Clin Invest 1993, 92:2746-2755

14. Carmeliet P, Schoonjans L, Kieckens L, Ream B, Degen J, Bronson R, De Vos R, van den Oord JJ, Collen D, Mulligan RC: Physiological consequences of loss of plasminogen activator gene function in mice. Nature 1994, 368:419-424

15. Berkenpas MB, Lawrence DA, Ginsburg D: Molecular evolution of plasminogen activator inhibitor-1 functional stability. EMBO J 1995, 14:2969-2977

16. Stefansson S, Petitclerc E, Wong MK, McMahon GA, Brooks PC, Lawrence DA: Inhibition of angiogenesis in vivo by plasminogen activator inhibitor-1. J Biol Chem 2001, 276:8135-8141

17. Tschanz SA, Burri PH, Weibel ER: A simple tool for stereological assessment of digital images: the STEPanizer. J Microscopy 2011, 243:47-59

18. Luyendyk JP, Cantor GH, Kirchhofer D, Mackman N, Copple BL, Wang R: Tissue factor-dependent coagulation contributes to alphanaphthylisothiocyanate-induced cholestatic liver injury in mice. Am J Physiol Gastrointest Liver Physiol 2009, 296:G840-G849

19. Laki K, Lorand L: On the solubility of fibrin clots. Science 1948, 108:280

20. Lorand L: A study on the solubility of fibrin clots in urea. Hung Acta Physiol 1948, 1:192-196

21. Kopec AK, Joshi N, Cline-Fedewa H, Wojcicki AV, Ray JL, Sullivan BP, Froehlich JE, Johnson BF, Flick MJ, Luyendyk JP: Fibrin(ogen) drives repair after acetaminophen-induced liver injury via leukocyte $\alpha \mathrm{M} \beta 2$ integrin-dependent upregulation of Mmp12. J Hepatol 2017, 66:787-797

22. Aleman MM, Byrnes JR, Wang JG, Tran R, Lam WA, Di Paola J, Mackman N, Degen JL, Flick MJ, Wolberg AS: Factor XIII activity mediates red blood cell retention in venous thrombi. J Clin Invest 2014, 124:3590-3600

23. Davydov L, Cheng JW: Tenecteplase: a review. Clin Ther 2001, 23: 982-997; discussion 1

24. Izumi S, Hughes RD, Langley PG, Pernambuco JR, Williams R: Extent of the acute phase response in fulminant hepatic failure. Gut 1994, 35:982-986 
25. Agarwal B, Wright G, Gatt A, Riddell A, Vemala V, Mallett S, Chowdary P, Davenport A, Jalan R, Burroughs A: Evaluation of coagulation abnormalities in acute liver failure. J Hepatol 2012, 57:780-786

26. Flick MJ, Du X, Degen JL: Fibrin(ogen)-alpha M beta 2 interactions regulate leukocyte function and innate immunity in vivo. Exp Biol Med 2004, 229:1105-1110

27. Draxler DF, Medcalf RL: The fibrinolytic system: more than fibrinolysis? Transfus Med Rev 2015, 29:102-109

28. Kang LI, Isse K, Koral K, Bowen WC, Muratoglu S, Strickland DK, Michalopoulos GK, Mars WM: Tissue-type plasminogen activator suppresses activated stellate cells through low-density lipoprotein receptor-related protein 1. Lab Invest 2015, 95:1117-1129

29. Yepes M, Sandkvist M, Moore EG, Bugge TH, Strickland DK, Lawrence DA: Tissue-type plasminogen activator induces opening of the blood-brain barrier via the LDL receptor-related protein. J Clin Invest 2003, 112:1533-1540

30. Wang H, Vohra BP, Zhang Y, Heuckeroth RO: Transcriptional profiling after bile duct ligation identifies PAI-1 as a contributor to cholestatic injury in mice. Hepatology 2005, 42:1099-1108
31. Holt MP, Cheng L, Ju C: Identification and characterization of infiltrating macrophages in acetaminophen-induced liver injury. J Leukoc Biol 2008, 84:1410-1421

32. Bergheim I, Guo L, Davis MA, Duveau I, Arteel GE: Critical role of plasminogen activator inhibitor-1 in cholestatic liver injury and fibrosis. J Pharmacol Exp Ther 2006, 316:592-600

33. Joshi N, Kopec AK, Towery K, Williams KJ, Luyendyk JP: The antifibrinolytic drug tranexamic acid reduces liver injury and fibrosis in a mouse model of chronic bile duct injury. J Pharmacol Exp Ther 2014, 349:383-392

34. Wang H, Zhang Y, Heuckeroth RO: PAI-1 deficiency reduces liver fibrosis after bile duct ligation in mice through activation of tPA. FEBS Lett 2007, 581:3098-3104

35. Beier JI, Guo L, Ritzenthaler JD, Joshi-Barve S, Roman J, Arteel GE: Fibrin-mediated integrin signaling plays a critical role in hepatic regeneration after partial hepatectomy in mice. Ann Hepatol 2016, 15 : $762-772$

36. Olson JD: D-dimer: an overview of hemostasis and fibrinolysis, assays, and clinical applications. Adv Clin Chem 2015, 69:1-46 